\title{
La comunicación institucional, epicentro de la empresa
}

\author{
Cristina BARANDA DEL CAMPO \\ Universidad Complutense de Madrid \\ cbcb.rm@gmail.com
}

Recibido: 06/07/2015

Aceptado: 12/09/2015

\section{RESUMEN:}

La información es el elemento primordial en una Institución, sea del carácter que sea. Fluye de cualquier departamento que la integra y esta información hay que recopilarla, ordenarla, sintetizarla, tratarla y por último y más relevante hay que distribuirla. No se puede retener ni ocultar, se debe canalizar para que todos los integrantes de esa empresa la conozcan, la entiendan, la asuman y la hagan suya. Es así como funcionan bien los organismos conociendo lo que trabajan, sus objetivos, sus límites, sus avances y sus logros. Los gabinetes de comunicación son el motor de la documentación. Gestionan los contenidos, crean archivos de datos y documentos y difunden la información, no solo entre los diferentes componentes de la empresa sino también entre los distintos estamentos de la sociedad, proyectando al exterior la imagen de la Institución para la que trabajan.

Palabras claves: Gabinete de prensa, gabinete de comunicación, comunicación institucional, información, datos, contenidos, canales de difusión, documentación y profesionales de la información.

\section{Institutional communication: the company epicenter}

\begin{abstract}
:
Information is the key element in an institution, whether the character is. Flows of any department that integrates and this information must be collected, we sort it, synthesize it, treat it and finally and most important have to distribute it. You cannot withhold or conceal, should be channeled to all members of that company know it, understand it, assume it and make it their own. This is how agencies work well knowing what work, its objectives, its limits, its progress and achievements. Communication offices are the engine of the documentation. Manage content, create data files and documents and disseminate information, not only between the different components of the company but also between different sectors of society, projecting abroad the image of the institution for which they work.
\end{abstract}

Keywords: Press office, communication office, institutional communication, information, dates, contents, dissemination channels, and documentation and information professionals.

\section{Referencia normalizada}

Baranda Campo del C. (2015). “La comunicación institucional, epicentro de la empresa”. Documentación de las Ciencias de la Información, Vol. 38: páginas. 143-151 


\section{INTRODUCCIÓN}

La aparición en las dos últimas décadas de numerosos profesionales dedicados a la comunicación no solo en los medios sino también en las empresas, bien directivos como departamentos enteros es un hecho. La información aumenta, la tecnología ayuda a ordenarla y canalizarla y el mundo empresarial se mentaliza de la importancia de tratarla y de comunicarla. Conllevar los métodos tradicionales con las nuevas tecnologías y solventar los problemas por gestión de contenidos.

Surgen diversas posibilidades para los profesionales de la información aunque tienen que competir con otros profesionales cualificados de la informática, la comunicación empresarial y del diseño de la Web e identidad corporativa, que les arrebatan tareas o al menos son una amenaza para ellos en lugar de un complemento o colaboración.

Han de estar integrados en la empresa para la que trabajan y saber organizar el conocimiento. Las nuevas tecnologías hacen que se desarrollen los métodos de gestión, organización y archivo y no por ello hay que quedarse atrás, sino todo lo contrario hay que ir con los tiempos incluso adelantarse a las necesidades ante la aglomeración de informaciones.

En los gabinetes de comunicación y en las diferentes empresas que tienen este órgano, la Documentación adquiere mayor protagonismo. Esto significa según el profesor Antonio García Jiménez “que los procedimientos documentales tienen un lugar en las operaciones y funciones comunicativas. Al mismo tiempo, representan un salto de calidad en lo referente a la mejora en el acceso a la información en el marco de la web y las intranet, para lo cual resultan beneficiosos los planteamientos de los que reivindican las ideas de capital Intelectual y de gestión del Conocimiento"1. Sus funciones documentales van desde la comunicación corporativa a la de relación con los medios

\section{LOS GABINETES DE COMUNICACIÓN}

Instituciones, empresas, movimientos sociales, partidos políticos, sindicatos, universidades, colegios profesionales, etc. dirigen cada vez más interés y recursos por la comunicación y se han dado cuenta de la necesidad de incorporar en su organigrama un gabinete o departamento que controle, analice, ejecuta y difunda las acciones de gestión de la comunicación transmitiendo la imagen positiva de la organización.

Los gabinetes de comunicación son "las fuentes activas, organizadas y habitualmente estables de información que cubren las necesidades comunicativas tanto internas como externas de aquellas organizaciones y/o personas de relieve que desean

${ }^{1}$ GARCÍA JIMÉNEZ, Antonio: El gabinete de prensa y comunicación como centro de documentación. (Madrid, 1999; pág.9) http://www.cobdc.org/jornedes/7JcD/78.pdf 
transmitir de sí mismas una imagen positiva a la sociedad influyendo de esta forma en la opinión pública”2.

El gabinete debe reflejar seguridad y coherencia, "tiene la función genérica de proyectar cohesión, de ofrecer sensación de que todo funciona y cada uno cumple su función dentro de la organización y, en definitiva, de transmitir una buena imagen global de la misma” 3. La estrategia de comunicación debe depender del órgano directivo y tiene que partir de una visión global de la comunicación pero al mismo tiempo ha de otorgar a la comunicación interna un papel primordial.

Además la comunicación interna es el sustento de la comunicación externa, porque todos y cada uno de los integrantes de la plantilla de la empresa o institución se convierten en transmisores de la imagen y son piezas de información hacia el exterior. La comunicación interna posibilita que los integrantes de una empresa se adapten a su funcionamiento "y es también el vehículo principal a través del cual los miembros de la agrupación pueden ayudar a dirigir cambios en la misma, influyendo en las actividades de otros individuos con los que cooperan" 4 .

En definitiva el gabinete de comunicación debe armonizar ambas comunicaciones interna y externa para hacerla realmente eficaz y debe relacionarse con los directores del resto de departamentos de la empresa, para que se canalice la información y llegue a todos.

\section{VÍAS DE COMUNICACIÓN GLOBAL}

La comunicación global da una imagen integrada de la institución. Por esta razón es importante que el gabinete esté estrechamente ligado al órgano ejecutivo de la empresa u organización y conocer su línea de actuación en cada momento, para poder organizar y transmitir la información que surja. "Si se hace depender de órganos intermedios, se pierde coordinación y se despilfarra tiempo y recursos humanos"5 Una jornada cualquiera de un profesional de la información consiste en comunicar, gestionar recursos, tratar con audiencias exigentes y en el ejercicio de esas tareas pueden encontrar problemas jurídicos o éticos, incluso políticos. Y en la facultad no se instruyen lecciones de destrezas y artes profesionales: comunicación, gestión, psicología y relaciones humanas, derecho institucional, deontología, etc.

\footnotetext{
${ }^{2}$ RAMÍREZ, Txema: Gabinetes de comunicación: funciones, disfunciones e incidencia. (Barcelona, 1995; pág.27) ISBN: 84-7676-311-5

${ }^{3}$ CAMACHO MARKINA, Idoia (2001): Comunicación Institucional. Funciones de los gabinetes de comunicación en la administración local. Revista Latina de Comunicación Social, 44

${ }^{4}$ LUCAS MARÍN, Antonio: La comunicación en la empresa y en las organizaciones. (Barcelona. Bosch; 1997; pág.164) ISBN84-7676-382-4

${ }^{5}$ RAMÍREZ, Txema: Gabinetes de comunicación: funciones, disfunciones e incidencia. (Barcelona, 1995; pág.27) ISBN: 84-7676-311-5
} 
La Universidad ilustra poco académicamente y lo indispensable para el desarrollo de la profesión hay que cultivarlo después. Un valioso tiempo se ha perdido durante esos años de formación, en los que las mentes están ávidas de aprendizaje y plenas de capacidad y no debería haber sucedido.

De algo debe servir la inexperiencia con la que salen multitud de profesionales, tras su formación universitaria. Es una llamada de atención, que año tras año se convierte en una situación de emergencia.

Cada día llegan a los medios de comunicación miles de noticias. Por no haber tiempo ni medios, no se pueden incluir todas, es en este punto donde los medios construyen la realidad que se nos muestra día a día y lo que hace distinto un medio de otro. Los medios cambian, pero también cambian los profesionales y sus roles. "Los profesionales de la comunicación deben ver en los gabinetes de prensa una nueva salida profesional"6. La información que sale desde los gabinetes de prensa de organizaciones y empresas es fidedigna, contrastada y bien elaborada. Son excelentes profesionales de la comunicación los que la producen y sin embargo están muy poco valorados.

\section{INFORMACIÓN INTERESANTE, COMPROMETIDA Y DIVULGADA}

Es la era de la comunicación en la que nos encontramos. La Sociedad de la información está integrada por autopistas, redes telemáticas, nuevas tecnologías, Internet, en los que la información es un valioso producto y su eje es la comunicación. Y las instituciones públicas no están aisladas de esta realidad, por lo que "no pueden vivir ajenas a este fenómeno que invita a manifestar la presencia de cada organismo en la sociedad y su porqué" 7 . Es un hecho que los colegios profesionales, las sociedades científicas y la generalidad de los colectivos sociales tienen tan poca resonancia en los medios de comunicación. Y que cuando se escucha algo de ellos, la mayoría de las veces es por algo negativo. Y está demostrado también que la información profesional ocupa muy poco espacio en los medios de información. ¿A qué se debe qué los periodistas tengan tan poco interés en la vida profesional? Los colegios profesionales y las empresas deben hacer mucho más por cambiar esta situación.

\footnotetext{
${ }^{6}$ TOMÁS FRUTOS, Juan y MARÍN CONESA, Ana Ma: Las relaciones con los medios: El funcionamiento de los gabinetes de prensa.

https://www.um.es/campusdigital/Cultural/gabinetes\%20prensa.htm

${ }^{7}$ SEBASTIÁN FERNÁNDEZ, Carmen: Principios generales de la comunicación en las organizaciones. Capital Humano, abril 1998, nº 110; pág. 44). ISSN: 1130-8117
} 


\section{INSTRUCCIONES DE ESTRATEGIA COMUNICACIONAL}

Son tres los pilares de una eficaz política de comunicación:

.- La relevancia de la información

Hay que conocer el medio en el que nos movemos. Tener toda la información, hay que averiguarla, investigarla y conocerla. Con todos los datos se consigue cualquier objetivo. La información es indispensable para afrontar adecuadamente cualquier problema. La información posee un potencial incalculable, del buen profesional depende su aprovechamiento y su máximo rendimiento. Nos movemos en la era digital. "La digitalización potencia las posibilidades de su explotación y hace más visible las diferencias entre instituciones"8

Los profesionales han de mentalizarse para lograr transformar la información en recurso estratégico. Para ello han de canalizar sus esfuerzos en potenciar el sitio web del organismo, afianzar el centro de documentación como motor que genera la energía documental y a la vez deben reforzar el fomento de la formación para el acceso a nuevas tecnologías, creando un portal con fácil acceso para todos.

.- La necesidad de utilizar bien la información y compartirla

Las organizaciones, empresas y colegios profesionales son colectivos que requieren información y que la manejan. Pero no basta con poseerla, hay que compartirla y canalizarla adecuadamente entre todos los interesados, para darse a conocer y conseguir transmitir su figura e imagen corporativa, en definitiva su identidad. Poseer toda la información es tener el poder del conocimiento, pero no se trata de acaparar el saber, se trata de difundirlo para que los integrantes del colectivo estén informados y constituyan esa identidad organizativa deseada.

-- La dificultad como posibilidad de éxito.

Las posibilidades de problemas son innumerables en toda organización. Un conflicto es un trance, pero puede y debe convertirse en una circunstancia positiva. La táctica empleada ante una situación crítica es lo que diferencia a una institución de otra. Y su plan de comunicación tiene mucho que ver o casi todo con la solución de su conflicto.

\footnotetext{
${ }^{8}$ RAMOS, Luis Fernando; DEL VALLE, Félix; MENDO, Concepción; TEJADA, Carlos y ARIAS, Alicia: Diseño de modelos para el análisis de la información en el sector público. (FESABID, Madrid, 15 de abril 2005; Grupo Publidoc UCM)

https://www.fesabid.org/madrid2005/descargas/.../comunicaciones/ramos_luis
} 


\section{LAS EMPRESAS, LOS COLEGIOS PROFESIONALES Y LA COMUNICACIÓN}

Los objetivos primordiales son informar hacia adentro, a los trabajadores y hacia fuera a los ciudadanos, la sociedad. Potenciar la imagen y la identidad de las empresas, ya que producir, produce cualquiera, pero hacerlo bien, no está al alcance de cualquiera. Proyectar la imagen corporativa que se desea depende de una estrategia comunicacional perfecta. Y para lograrla hay que trabajar estrechamente con los medios colaborando con ellos conocerlos.

Las empresas, las Instituciones y los colegios profesionales deben desarrollar un plan de comunicación elaborado a la medida de sus necesidades. Su función principal es defender a los profesionales y defender a la sociedad. La unión profesional produce la fuerza de las instituciones y hay que saber aprovecharla para lograr los fines propuestos. Por este motivo, la estrategia de comunicación que se despliegue tendrá mucho que ver con el éxito o fracaso del plan de objetivos trazado.

\section{CON OTRA MENTALIDAD COMUNICATIVA: INTEGRADORA Y EXPANSIVA}

Cambiar de aires, reforzar las estrategias, incluso en ocasiones partir de cero, puede traer consigo nuevos tiempos y muchas posibilidades de éxito en una empresa o institución. Sin duda la comunicación que se lleve a cabo se convertirá en la llave del logro. Hay que crear valor e implicar a los profesionales para que colaboren. La Institución se reinventa como fuente de información y como gestor de servicios que el empresario ha de valorar.

En un Gabinete de Comunicación fluye la información y se debe ordenar, tratar y elaborar su contenido. Desde este epicentro de la empresa que es el gabinete se han de organizar congresos, jornadas y cursos. Se ha de editar una revista corporativa, pero ha de ser atractiva, que no solo interese a su colectivo, sino que lleve secciones que enganchen a otros ámbitos. Los comunicadores han de organizar una web que ofrezca información de interés y servicios interactivos, válidos, prácticos y actualizados. La web que diseñen debe ser un lugar de encuentro para todos y debe mostrar una verdadera identidad corporativa. Otros elementos básicos pero no menos importantes, como el correo electrónico, las bases de datos, la Intranet, los buzones con consejos prácticos, los mensajes a los trabajadores, etc., son cuidados, mimados y tratados por los profesionales de la comunicación.

\section{LA DEMANDA DEL CLIENTE DE UNA EMPRESA O INSTITUCIÓN}

Lo buscado, lo ofrecido y lo solicitado por los clientes nuevos, y por los clientes antiguos que guardan una exquisita fidelidad, son servicios que sólo las empresas les pueden entregar. Estos clientes son profesionales que tienen la oportunidad de mani- 
festar lo que quieren y de presentarse en la sociedad a través de los medios y que les vean como quieren mostrarse. Por eso es imprescindible profesionalizar la gestión de todo ello, del marketing, de la publicidad, de las relaciones públicas y de la comunicación.

\section{TRABAJAR PARA EL ENTORNO Y HACIA EL EXTERIOR.}

Los profesionales elaboran el plan, y para ello definen objetivos comunicacionales a corto, medio y largo plazo. Han de estudiar los medios, conocerlos y crear con ellos un pacto de colaboración. Ofreciendo noticias, siendo válidos, creando complicidad y elementos comunes.

"Las instituciones públicas tienen obligación de comunicarse con la sociedad y, por tanto, de producir mensajes específicos dirigidos a sus diferentes públicos. En este sentido, una inadecuada información es una importante barrera para el mejor funcionamiento de la administración, de modo que la eficacia de una Institución acabará dependiendo de su política comunicativa”9

Es fundamental en la empresa y las instituciones tener portavoces y productores autorizados de opinión, expertos en el arte de la prudencia y la valentía.

Los miembros de un gabinete de comunicación han de fortalecer la imagen de la institución para la que trabajan y estrechar la relación con otras instituciones. Para conseguirlo definen unas estrategias y organizan actividades sociales en las que están presentes.

\section{ELABORAR UN PROTOCOLO DE COMUNICACIÓN}

Son los comunicadores los que han de elegir el procedimiento y las estrategias de comunicación para dar a conocer la empresa en el ámbito institucional primero, y proyectar la imagen deseada al exterior después, para conseguir unos objetivos que han de definirse desde el inicio del protocolo comunicacional.

Hay que saber el momento adecuado para cada información que se transmite y medir cuando hay que guardar silencio, ya que a veces no resulta rentable. La callada por respuesta no es la mejor estrategia ni la opción más aconsejable. El silencio no produce efectos positivos casi nunca.

La comunicación se aprende, se practica y se mejora. En la actualidad se valora a las personas, a las empresas y a las instituciones, no solo por lo que hacen sino también por la imagen que la audiencia se hace de ellas.

${ }^{9}$ DEL CASTILLO HERMOSA, Jaime; BAYÓN ESTEBAN, Ma Mercedes y ARTETA ARRÚE, Rosa: La empresa ante los medios de comunicación. (Bilbao, Deusto. 1992; pág. 122) ISBN: 84-234-1075-7 
Ningún profesional puede permitirse el lujo de ignorar la imagen que proyecta. Conseguir transmitir una imagen idónea a los medios es un objetivo primordial e imprescindible.

Los comunicadores tenemos una oportunidad única de influir en la sociedad y de crear tendencias, pero esto supone una gran responsabilidad añadida a nuestra tarea de informar. Hemos de ser conscientes, muy conscientes de lo que hacemos y decimos pues esos contenidos que emitimos pueden acarrear unas u otras consecuencias.

"Ninguna entidad importante existe hoy sin preocuparse de la comunicación, porque ésta se ha revelado como indispensable en un universo competitivo. El éxito de una institución, de un político, de un producto, está relacionado en proporción directa a la eficacia de su actividad comunicativa (...) Y no cabe otra alternativa que comunicar. Cualquier entidad que intente sobrevivir dignamente ha de emitir mensajes a los ciudadanos, para vender sus productos o servicios”10

\section{CONCLUSIONES}

La información que sale desde los gabinetes de prensa de organizaciones y empresas es fidedigna, contrastada y bien elaborada. Son excelentes profesionales de la comunicación los que la producen y sin embargo están muy poco valorados.

Los profesionales han de mentalizarse para lograr transformar la información en recurso estratégico. Para ello han de canalizar sus esfuerzos en potenciar el sitio web del organismo, afianzar el centro de documentación como motor que genera la energía documental y a la vez deben reforzar el fomento de la formación para el acceso a nuevas tecnologías, creando un portal con fácil acceso para todos.

Poseer toda la información es tener el poder del conocimiento, pero no se trata de acaparar el saber, se trata de difundirlo para que los integrantes del colectivo estén informados y constituyan esa identidad organizativa deseada.

La unión profesional produce la fuerza de las instituciones y hay que saber aprovecharla para lograr los fines propuestos. Por este motivo, la estrategia de comunicación que se despliegue tendrá mucho que ver con el éxito o fracaso del plan de objetivos trazado.

Es fundamental en la empresa y en las instituciones tener portavoces y productores autorizados de opinión, expertos en el arte de la prudencia y la valentía.

${ }^{10}$ ÁLVAREZ DOMÍNGUEZ, Tomás y CABALLERO HUESO, Mercedes: Vendedores de imagen: los retos de los nuevos gabinetes de comunicación. (Barcelona. Paidós; 1997; pág. 19-20) ISBN: 84-4930370-2. 


\section{BIBLIOGRAFÍA}

ÁLVAREZ DOMÍNGUEZ, Tomás y CABALLERO HUESO, Mercedes: Vendedores de imagen: los retos de los nuevos gabinetes de comunicación. (Barcelona. Paidós; 1997) ISBN: 84-493-0370-2.

CAMACHO MARKINA, Idoia (2001): Comunicación Institucional. Funciones de los gabinetes de comunicación en la administración local. Revista Latina de Comunicación Social, 44.http://www-ulles/publicaciones/latina/2001/latina44septiembre/4403camacho.htm

DEL CASTILLO HERMOSA, Jaime; BAYÓN ESTEBAN, MªMercedes y ARTETA ARRÚE, Rosa: La empresa ante los medios de comunicación. (Bilbao, Deusto. 1992) ISBN: 84-234-1075-7.

GARCÍA JIMÉNEZ, Antonio: El gabinete de prensa y comunicación como centro de documentación. (Madrid, 1999) http://www.cobdc.org/jornedes/7JcD/78.pdf

GUARDIA JIMÉNEZ, Alfredo: Profesionalización de los gabinetes de comunicación. (9 y 10 julio 2015)

http://www.upo.es/olavideencarmona/exportsites/upo.../2015/2015cc09.pdf

LUCAS MARÍN, Antonio: La comunicación en la empresa y en las organizaciones. (Barcelona. Bosh; 1997). ISBN84-7676-382-4.

RAMÍREZ, Txema: Gabinetes de comunicación: funciones, disfunciones e incidencia. (Barcelona, 1995) ISBN: 84-7676-311-5

RAMOS, Luis Fernando; DEL VALLE, Félix; MENDO, Concepción; TEJADA, Carlos y ARIAS, Alicia: Diseño de modelos para el análisis de la información en el sector público. (FESABID, Madrid, 15 de abril 2005; Grupo Publidoc UCM)

https://www.fesabid.org/madrid2005/descargas/.../comunicaciones/ramos_luis

SEBASTIÁN FERNÁNDEZ, Carmen: Principios generales de la comunicación en las organizaciones. Capital Humano, abril 1998, n 110; pág. 44-46). ISSN: 11308117.

TOMÁS FRUTOS, Juan y MARÍN CONESA, Ana Ma: Las relaciones con los medios: El funcionamiento de los gabinetes de prensa.https://www.um.es/campusdigital/Cultural/gabinetes\%20prensa.htm 